\title{
Cáncer de mama triple negativo: terapias sistémicas actuales y experiencia local
}

\author{
Glomaryeth Luque S. ${ }^{1}$, Benjamin Walbaum G. ${ }^{2}$, Mauricio Camus A. ${ }^{1}$, Francisco Domínguez C. ${ }^{1}$, \\ Tomas Merino L. ${ }^{2}$, Francisco Acevedo C. ${ }^{2}$ y César Sánchez R. ${ }^{2}$
}

'Departamento de Cirugía

Oncológica y Maxilofacial. Facultad de Medicina,

Pontificia Universidad Católica

de Chile. Santiago, Chile.

2Departamento de Hemato-

Oncología. Facultad

de Medicina, Pontificia

Universidad Católica de Chile. Santiago, Chile.

Recibido 2020-07-06 y aceptado 2020-08-19

Correspondencia a: César Sánchez R. cgsanchezr@gmail.com

\section{Triple negative breast cancer: current systemic therapy and local experience}

Breast cancer is the leading cause of cancer death in Chilean women and worldwide. It is a heterogeneous disease and four different subtypes have been identified based on clinical, histological and molecular features, which correlate with different treatment tumor sensitivity. Triple negative breast cancer is characterized by its aggressiveness, early relapse, and a greater tendency to present in advanced stages. It frequently affects young women, with cancer family history, especially breast or ovarian cancer. The approved systemic therapy for triple negative breast cancer is chemotherapy; however, recently, targeted therapies with checkpoint inhibitors and polyadenosine diphosphate ribose polymerase inhibitors have been shown to be effective in selected patients and have been added to the therapeutic arsenal for triple negative breast cancer. Given the appearance of these new strategies, it seems relevant to understand the heterogeneity of this disease, the mechanisms of action behind new therapies, clinical results, and the criteria to select patients for molecular therapies. We present a review of the current systemic therapy of this breast cancer subtype. Key words: breast cancer; triple negative; immunotherapy; BRCA; PARP inhibitors.

\section{Resumen}

El cáncer de mama $(\mathrm{CM})$ es la principal causa de muerte por cáncer en mujeres chilenas. Es una enfermedad heterogénea, en la cual se han identificado cuatro subtipos básicos, determinados según características clínicas, histológicas y moleculares, los que se relacionan a estrategias terapéuticas. El CM triple negativo (CMTN) se caracteriza por su agresividad, recaída temprana y mayor tendencia a presentarse en etapas avanzadas. Frecuentemente afecta a mujeres jóvenes o con antecedentes familiares de CM u ovario. La única terapia sistémica aprobada para el CMTN es la quimioterapia; sin embargo, recientemente terapias moleculares con inhibidores de puntos de control inmune e inhibidores de la poli-adenosina difosfato ribosa polimerasa, han mostrado eficacia en pacientes seleccionados, y se han agregado al arsenal terapéutico para CMTN. Dada la aparición de estas nuevas estrategias, parece relevante entender la heterogeneidad de esta enfermedad, los mecanismos de acción de las nuevas terapias, resultados clínicos y criterios de selección de pacientes para terapias moleculares. Presentamos una revisión de la terapia sistémica actual del CMTN.

Palabras clave: cáncer de mama; cáncer de mama triple negativo; inmunoterapia; BRCA; inhibidores PARP.

\section{Introducción}

Estimaciones del año 2018 indican que 5.400 chilenas fueron diagnosticadas con cáncer de mama (CM) y 1.700 murieron por la enfermedad; siendo, por tanto, la principal causa de muerte por cáncer en mujeres en nuestro país ${ }^{1}$. Es una enfermedad heterogénea, donde se han identificado al menos 4 subtipos diferentes, basados en sus características clínicas, histológicas y moleculares (Tabla 1) ${ }^{2}$. Aquellos CM que no expresan el receptor de estrógenos (RE), progesterona (RP) y tampoco sobreexpresan/amplifican el receptor del factor de crecimiento epidérmico humano tipo 2 (HER2), se denominan triples negativos (TN). El cáncer de mama triple negativo (CMTN) presenta frecuentemente una elevada tasa de proliferación, alteración en genes de reparación del ADN y ma- 
Tabla 1. Clasificación patológica y molecular, pronóstico y tratamiento por subtipo del cáncer de mama

\begin{tabular}{|lcccc|}
\hline & Luminal A & Luminal B & HER2-enriquecido & Triple Negativo \\
Característica molecular & RE+/HER2- & RE+/HER2+/- & HER2+ & RE-/RP-/HER2- \\
Definición patológica & RE $+\geq 1 \%$ & RE+ $\geq 1 \%$ & HER2 IHQ: 3+ o FISH + & RE-/RP-/HER2- por IHQ \\
Molécula efectora & RE $\alpha$ & RE $\alpha$ & HER2 & Desconocido \\
Niveles de Ki67 & Bajo & Alto & Alto & Alto \\
\% de casos & $45 \%$ & 35 & $15-20 \%$ & $10-15 \%$ \\
SG 5 años (etapa I) & $\sim 99 \%$ & $>90 \%$ & $\sim 94 \%$ & $25 \%$ \\
SG (etapa IV) & $4-5$ a. & $2-3$ a. & QT + Anti-Her2 & QT+ inmunoterapia \\
Terapia sistémica & TE & TE/QT & a. & \\
\hline
\end{tabular}

Abreviaciones: RE: receptor estrógeno; HER2: receptor para el factor de crecimiento epidérmico tipo 2; RP: receptor progesterona; IHQ: inmunohistoquímica; FISH: hibridación in situ con inmunofluorescencia; Ki67: antígeno de proliferación celular; SG: sobrevida global; TE: terapia endocrina; QT: quimioterapia.

yor inestabilidad genómica ${ }^{3}$. Ello le confiere mayor sensibilidad a la quimioterapia (QT), a drogas que tienen como blanco defectos en la reparación del DNA (inhibidores de la poli-adenosina difosfato ribosa polimerasa: PARPi) y a inhibidores de puntos de control (checkpoint) inmunes. Recientemente, se han incorporado a la QT convencional nuevas estrategias de manejo sistémico, que incluyen drogas con blancos moleculares y que requieren para su indicación la determinación de biomarcadores ${ }^{4}$. Presentamos una revisión del tema, enfocada en las características del CMTN, la evidencia que sustenta el manejo sistémico actual de este subtipo y una reflexión de los desafíos en nuestro país con respecto a su caracterización y manejo.

\section{Materiales y Método}

Junto a una breve revisión narrativa, revisamos la evidencia actual de la terapia del CMTN. Realizamos una búsqueda en PUBMED, con los términos "triple negative", "breast cancer", excluyendo CM "HER2 positive" y "hormone-dependent". La última búsqueda se hizo el 02-05-2020 con los filtros: "clinical trial", "humans" $y$ "published in the last 5 years". Aplicando estos filtros se obtuvo 132 citaciones, de las cuales 27 eran estudios clínicos fase III en pacientes con CMTN.

Extracción de datos en artículos obtenidos de la revisión de PUBMED

Se obtuvo: número de pacientes incluidos, tratamiento y escenario del tratamiento, resultados en sobrevida libre de progresión (SLP) y sobrevida global (SG).

\section{Aspectos generales del CMTN}

En los registros poblacionales de cáncer no existen datos acerca de la incidencia de CMTN a nivel nacional. Hemos reportado que el $11 \%$ de nuestros pacientes con CM presentan este subtipo (Figura $1)^{5}$; por tanto, estimamos que aproximadamente 600 mujeres serán diagnosticadas con CMTN anualmente en Chile.

El subtipo TN se ha descrito con mayor frecuencia en mujeres jóvenes, con historia familiar de síndromes asociados a $\mathrm{CM}$ u ovario, y en latinos y afroamericanos. Se asociaría a obesidad, en particular en mujeres premenopáusicas; nuliparidad y ausencia de lactancia ${ }^{6,7}$.

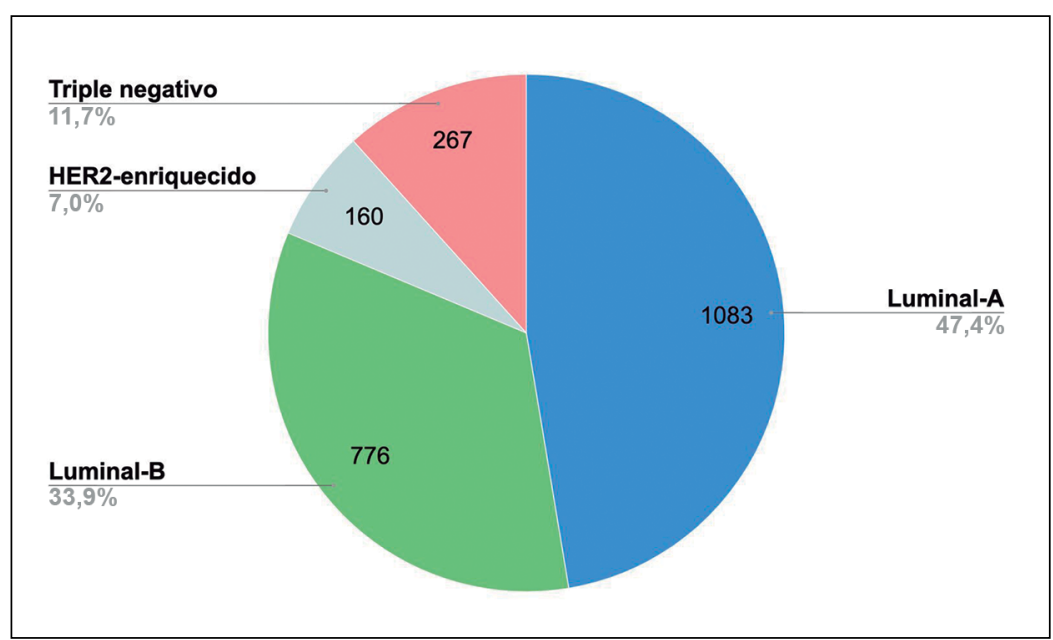

Figura 1. Distribución de cáncer de mama por subtipo en una cohorte de pacientes chilenas en una institución universitaria (1997-2018). Abreviaciones: HER2: receptor del factor de crecimiento epidérmico humano tipo 2. 
Se caracteriza por presentar recaídas tempranas (habitualmente antes de los primeros 5 años tras el diagnóstico) y mayor tendencia a debutar en etapas avanzadas, principalmente con metástasis viscerales $^{8}$. La Figura 2 muestra la SG a 5 años reportada por nuestro grupo, según etapa de la enfermedad ${ }^{5}$.

La definición clásica del CMTN implica ausencia de expresión, a través de inmunohistoquímica, del RE, RP y de HER2. Histológicamente, es una neoplasia pobremente diferenciada, de alta proliferación celular y tasa mitótica $y$, al igual que otros subtipos, heterogénea, incluyendo subconjuntos de pronósticos variables ${ }^{9}$. Una forma de subdividirse utilizando inmunohistoquímica es en CMTN basales y no basales; los primeros caracterizados por la expresión de citoqueratinas (CK) 5/6 y del receptor

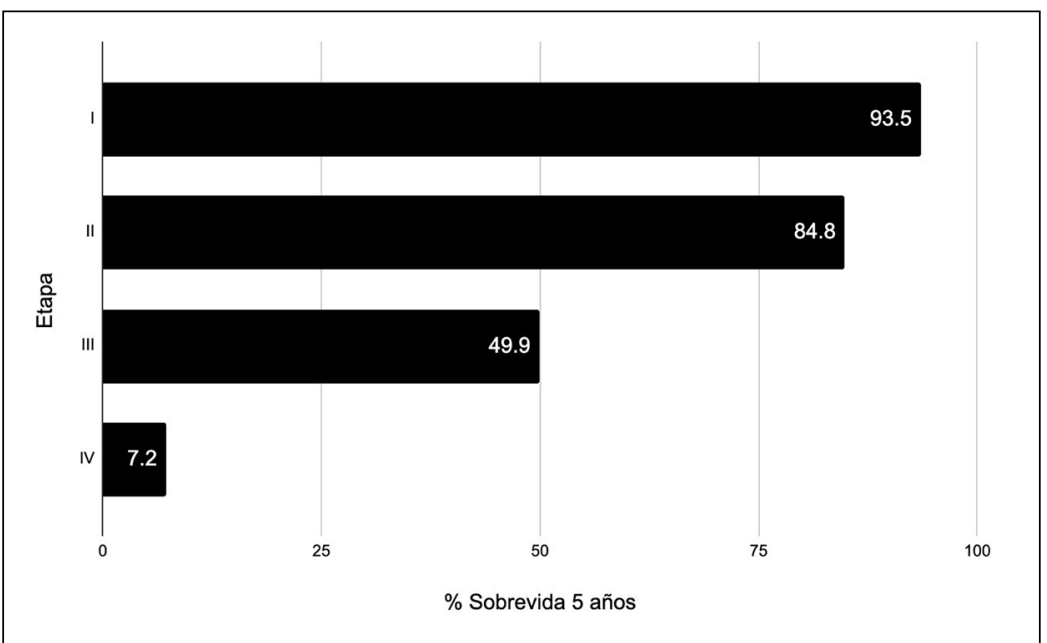

Figura 2. Sobrevida global a 5 años en pacientes chilenas con cáncer de mama triple negativo según etapa. del factor de crecimiento epidérmico humano tipo 1 $\left(\right.$ EGFR 1) ${ }^{10}$. La determinación de su perfil molecular intrínseco ha distinguido más grupos; algunos con un patrón similar a tumores hormono-dependientes y otros que expresan el receptor de andrógenos, por ejemplo. No obstante, esta clasificación por subtipos moleculares aún no es aplicable enteramente a la práctica clínica en nuestra realidad, al no tener un correlato terapéutico definido y reservado por ahora, en muchos casos, a su aplicación en ensayos clínicos

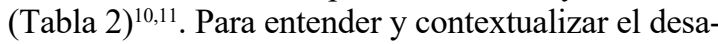
rrollo de terapias moleculares recientes en CMTN, revisaremos algunos aspectos generales de biomarcadores asociados a su clasificación y respuesta a terapias sistémicas.

\section{Biomarcadores asociados a CMTN y terapias moleculares}

$C K$ : Las células de la capa externa, a lo largo de la membrana basal del epitelio de los conductos de la glándula mamaria, son reactivas a CK5 y CK6. Su expresión permite por tanto determinar CMTN con fenotipo basal (CK5/6+ y EGFR 1+) y no basal ${ }^{12}$. El estudio CREATE X mostró beneficio en la SG para el uso de capecitabina post QT neoadyuvante (QTNA) en pacientes con enfermedad residual, particularmente para el subgrupo $\mathrm{TN}^{13}$. El grupo español GEICAM mostró beneficio de esta estrategia sólo para aquellos CMTN con fenotipo no basal ${ }^{14}$.

Receptor de andrógenos: Su expresión es variable. Una revisión sistemática reciente mostró que se expresa en un $28 \%$ de los CMTN, sin guardar correlación con $\mathrm{SG}$ o libre enfermedad ${ }^{15}$. La terapia con antiandrógenos es una opción en CMTN avanzado ${ }^{16}$.

Factor de crecimiento endotelial vascular $(V E G F)$ : La alteración en la angiogénesis es una

Tabla 2. Terapias dirigidas según subtipo de cáncer de mama triple negativo

\begin{tabular}{|lll|}
\hline Subtipo de CMTN & Vías alteradas & Terapias \\
\hline Basal & Reparación de ADN & PARPi, platinos \\
Mesenquimal & PI3K/AKT/mTOR, MET, TGF-B, NOTCH & TKI \\
\hline RAL & RA & Antiandrógenos \\
Inmunomodulador & Señalización de la inmunidad & Inhibidores de checkpoint \\
HER2 enriquecido & HER2 & Vacunas \\
\hline
\end{tabular}

Abreviaciones: CMTN: cáncer de mama triple negativo; PARPi: inhibidores de poli-adenosina difosfato ribosa polimerasa; PI3K: fosfatidilinositol 3 quinasa; AKT: proteína quinasa B; mTOR; complejo sensible a rapamicina; MET: transición epitelio-mesénquima; TGF-B: factor de crecimiento transformante b; NOTCH: receptor transmembrana de comunicación yuxtacrino; TKI: inhibidor de tirosina quinasa; RAL: receptores de andrógenos luminal; RA: receptor de andrógenos; HER2: receptor para el factor de crecimiento epidérmico tipo 2. 
parte central en la carcinogénesis de las neoplasias en general. Ensayos clínicos usando un anticuerpo contra VEGF en combinación con QT han mostrado beneficios en las tasas de respuesta y SLP de pacientes con $\mathrm{CMTN}^{17}$. Su uso está limitado dada la ausencia de datos que muestren impacto en $\mathrm{SG}$, sumado a la falta de biomarcadores predictivos para seleccionar pacientes.

Receptor de muerte programada (PD1) y ligando del receptor de muerte programada (PDL-1): PDL1 y PD1 son proteínas que actúan como puntos de control (checkpoint) inmunes. Tanto PDL-1, presente principalmente en células neoplásicas, como PD1 en linfocitos T, ayudan a mantener el equilibrio y control del sistema inmune. La unión de PDL-1 a PD1 impide la activación de células $\mathrm{T} y$, por ende su acción citotóxica sobre células neoplásicas (Figura 3). Por tanto, la inhibición farmacológica de esta unión por medio de inhibidores de estos puntos de control inmune, utilizando anticuerpos anti-PDL-1 $\mathrm{y}$ anti-PD1, permite que el propio sistema inmune destruya las células tumorales. Aproximadamente el $40 \%$ de los CMTN avanzados expresan PDL-1 (en células no tumorales) y serían candidatos para el uso de inhibidores de checkpoint inmunes, con beneficios en $\mathrm{SG}^{18,19}$.

Infiltración de linfocitos (TIL): Tumores con predominio de linfocitos, definidos como más del $60 \%$ de infiltración estromal o tumoral, tienen tasas de respuesta patológica completa (RPC) de 41,7\% en comparación con un $2 \%$ para tumores sin TIL. También es un marcador de sensibilidad a inhibidores de checkpoint inmunes.

Mutación BRCA: Los genes BRCA participan de los mecanismos de reparación del ADN. Del total de pacientes con CMTN, hasta un $20 \%$, tendrían mutaciones germinales de genes $B R C A$, por lo tanto, serían candidatos para el uso de PARPi. PARP es una enzima que participa en otros mecanismos, diferentes a BRCA, para reparar el ADN. La inhibición de PARP, por tanto, es particularmente efectiva en neoplasias que tienen alteraciones de la reparación del ADN por otros mecanismos PARP independientes, como son aquellas células con mutaciones de $B R C A$ (Figura 4). En Chile, la frecuencia de mutaciones de $B R C A 1$ y $B R C A 2$ en pacientes con $\mathrm{CM}$ hereditarios, varía entre $15 \%$ al $20 \%$, con un predominio de $B R C A 1^{20}$.

A pesar de todos estos biomarcadores y, a diferencia del CM hormono-dependiente, que tiene un

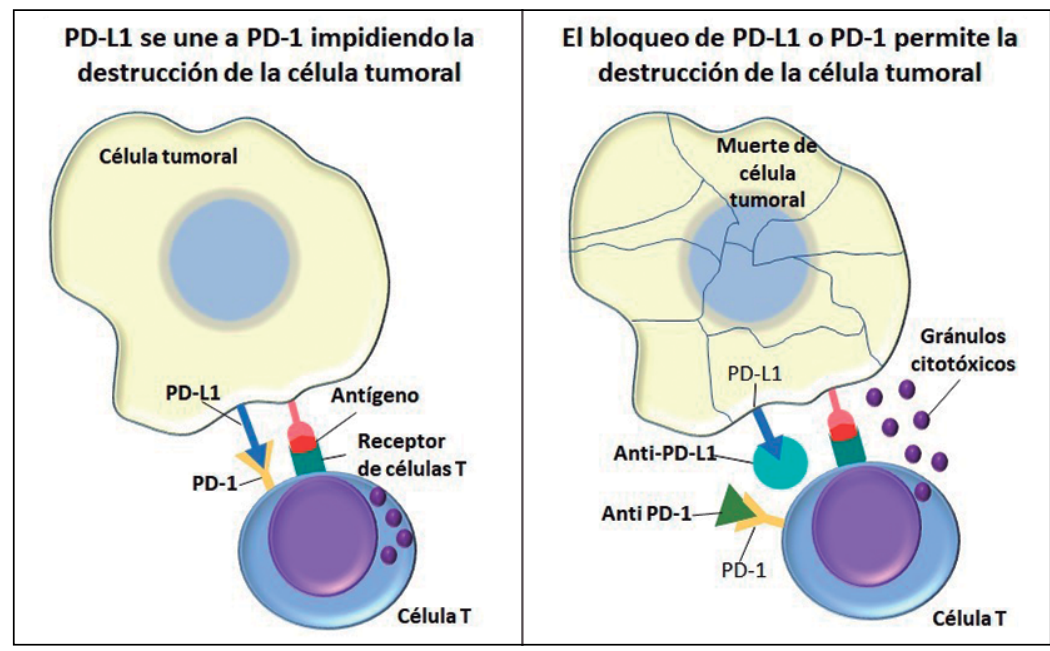

Figura 3. Mecanismo acción de inhibidores de Checkpoint PD1 y PDL1. Anticuerpos anti PD1 y PDL1 permiten acción antitumoral de linfocitos T CD8 + mediante la liberación de gránulos citotóxico. Abreviaciones: PD1: receptor de muerte programada; PDL-1: ligando del receptor de muerte programada.

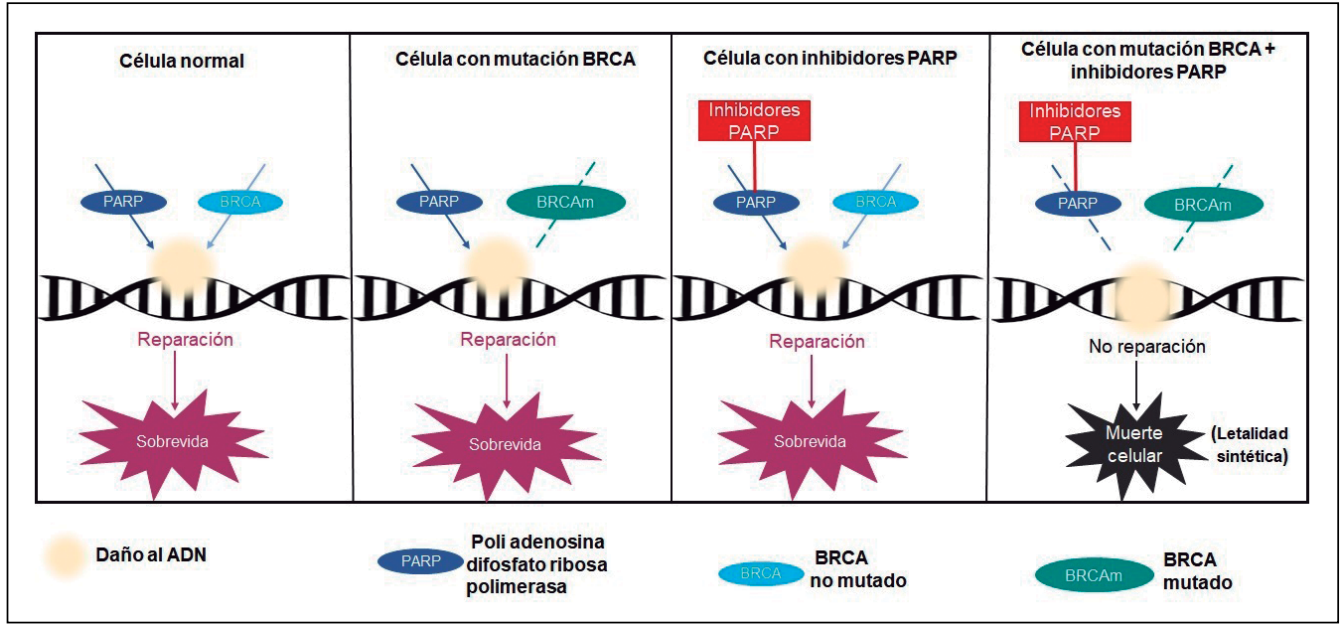

Figura 4. Mecanismo acción de inhibidores PARP (poli-adenosina difosfato ribosa polimerasa) 
Tabla 3. Estudios fase II, uso de platino neoadyuvante en cáncer de mama triple negativo con análisis de sobrevida

\begin{tabular}{|c|c|c|c|c|c|}
\hline \multirow[t]{2}{*}{ Estudio } & \multirow[t]{2}{*}{$\mathbf{n}$} & \multirow[t]{2}{*}{ Ramas } & \multirow[t]{2}{*}{ RPC (\%) } & \multicolumn{2}{|c|}{ Sobrevida (HR) } \\
\hline & & & & SLE & SG \\
\hline GeparSixto $^{22}$ & 315 & $\begin{array}{c}\mathrm{QT} \\
\mathrm{QT}+\mathrm{Cb}\end{array}$ & $\begin{array}{l}37 \\
53\end{array}$ & $\begin{array}{c}0,56(0,34-0,93) \\
p=0,022\end{array}$ & $0,60(0,32-1,12)$ \\
\hline $\mathrm{CALGB}$ ALLIANCE ${ }^{23}$ & 218 & $\begin{array}{c}\mathrm{QT} \\
\mathrm{QT}+\mathrm{Cb}\end{array}$ & $\begin{array}{l}41 \\
54\end{array}$ & $\begin{array}{c}0,84 \\
(0,58-1,22)\end{array}$ & $\begin{array}{c}1,15 \\
(0,74-1,79)\end{array}$ \\
\hline
\end{tabular}

Abreviaciones: CMTN: cáncer de mama triple negativo; RPC: respuesta patológica completa; QT: quimioterapia; $\mathrm{Cb}$ : carboplatino; SG: sobrevida global; SLE: sobrevida libre de enfermedad; HR: Hazard ratio.

marcador específico, predictor de respuesta como es el RE; en la actualidad, la mayoría de los pacientes con CMTN requieren el uso de QT, tanto para el tratamiento de la enfermedad localizada, como en el tratamiento con intención paliativa en etapas avanzadas.

\section{Estrategias actuales de manejo sistémico en cáncer de mama triple negativo}

\section{Quimioterapia neoadyuvante}

La QT es el tratamiento sistémico aprobado actualmente para el CMTN localizado. Incluye diferentes agentes y formas de administración, pero que no difieren, en la estructura general, de aquella utilizada para otros subtipos. Excepciones serían mayor sensibilidad a la adición de platinos y a la densidad de dosis (menor intervalo de tiempo entre dosis de QT). Pacientes con baja carga de

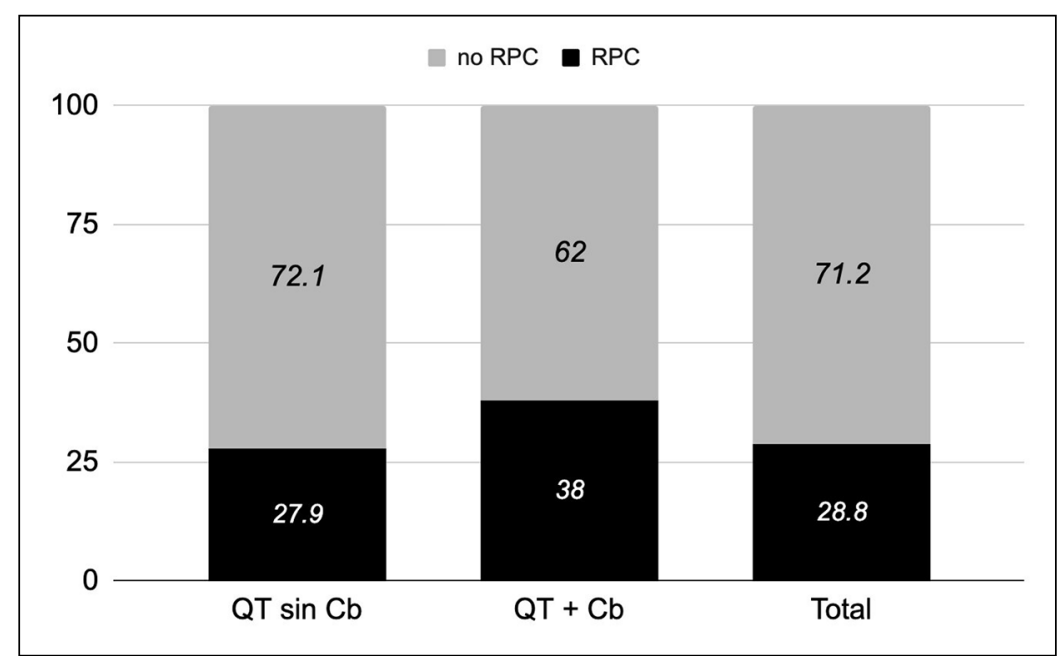

Figura 5. Tasa de respuesta patológica completa en cáncer de mama triple negativo según esquema de quimioterapia. Abreviaciones: CMTN: cáncer de mama triple negativo; RPC: respuesta patológica completa; QT: quimioterapia; Cb: carboplatino. enfermedad y, por tanto, bajo riesgo de enfermedad metastásica (tumores $<0,5 \mathrm{~cm}$, sin compromiso linfonodal: T1aN0), además de pacientes que no toleren el tratamiento, podrían no beneficiarse de la terapia sistémica citotóxica ${ }^{21}$.

Dada la frecuente alteración en los mecanismos de reparación del ADN presentes en CMTN, se ha demostrado que el uso de platinos (cisplatinocarboplatino) aumenta la tasa de RPC cuando se usa como complemento en neoadyuvancia, aunque sin un efecto significativo y consistente en la SG (Tabla 3$)^{22,23}$. En nuestra experiencia, en 192 pacientes con CMTN, aquellos que recibieron carboplatino (Cb) neoadyuvante obtuvieron una tasa de RPC mayor, pero no estadísticamente significativa $(38 \%$ vs $28 \% \mathrm{p}=0,46$ ) (Figura 5). La obtención de RPC, definida como la desaparición del tumor invasivo en la biopsia quirúrgica post QTNA, se ha asociado con un aumento en SG, lo que permite utilizarla como un marcador sustituto de $\mathrm{SG}^{24}$. Pacientes que no logran RPC, serían potenciales candidatos al uso de capecitabina adyuvante ${ }^{13}$. El uso de QTNA hoy en día permite no sólo obtener información pronóstica, sino también abrir una ventana terapéutica en la adyuvancia, según los resultados obtenidos en la biopsia quirúrgica ${ }^{25}$.

\section{Quimioterapia en CMTN avanzado}

De acuerdo a nuestros datos un 7\% de los CMTN debuta con enfermedad metastásica y un $20 \%$ de los pesquisados en etapas precoces recurrirá a distancia, la gran mayoría antes de 5 años $^{5,26}$. La mediana de SG en CMTN avanzado es de 19 meses en nuestros registros, muy por debajo de las sobrevidas que vemos en CM avanzados luminales o HER2+ (Tabla 1). Mientras no existe un esquema definido de QT paliativa para estos casos, guías clínicas sugieren, dependiendo de las terapias usadas en adyuvancia, antraciclinas o taxanos como monodroga en primera línea ${ }^{21}$, reservando QT combinada para pacientes 
con alta carga tumoral o en que busquemos rápida respuesta, sin diferencias en SG al comparar con monoterapia. En caso de pesquisar mutaciones $B R C A$, el uso de platinos ha mostrado tasas de respuesta superiores a taxanos ${ }^{27}$.

\section{Terapias moleculares aprobadas en cáncer de mama triple negativo}

Dada las características biológicas ya descritas del CMTN, principalmente la alteración en mecanismos de reparación del ADN y aumento en la inmunogenicidad por expresión de neoantígenos, se han desarrollado terapias dirigidas a esas características particulares. Los inhibidores de puntos de control (checkpoint) inmunes y PARPi han demostrado eficacia en pacientes seleccionados ${ }^{18,28,29}$.

El estudio Keynote 522 evaluó la adición de pembrolizumab, un anticuerpo monoclonal anti PD1, a un régimen de QTNA que incluía platinos. El análisis inicial mostró una tasa de RPC más alta con la adición de pembrolizumab, y en el análisis interino, un beneficio en sobrevida libre de enfermedad (SLE) independiente de la expresión de PDL- ${ }^{30}$ (Tabla 4).

En el contexto de CMTN avanzado, atezolizumab (otro inhibidor de checkpoint inmunológico) asociado a taxanos, ha demostrado beneficio en SG en pacientes con CMTN que tienen expresión de PDL-1 en células no tumorales $>1 \%{ }^{19}$. Finalmente, pembrolizumab combinado con QT en primera línea, mostró una mejora significativa en SLP frente a QT sola en pacientes con CMTN avanzado, con expresión PDL-1 determinada por un método llamado CPS (combined positive score $>10)(\text { Tabla } 4)^{31}$.

El uso de PARPi ha mostrado en dos estudios fase III incrementar la SLP de pacientes CMTN avanzado $B R C A m$ vs QT diferentes a platinos ${ }^{32,33}$; sin beneficio en etapa temprana ${ }^{34}$ (Tabla 5).

Estas estrategias confirman la importancia del estudio de marcadores predictivos para nuestras pacientes, como la expresión de PDL-1 y mutaciones en la línea germinal de BRCA1 o BRCA2.

Nuevas terapias con anticuerpos conjugados también aparecen en el horizonte. El Sacituzumab govitecan-hziy conjuga un anticuerpo monoclonal humanizado anti-trofoblasto (Trop2), proteína transmembrana sobre-expresada en CMTN, con SN-38, este último un metabolito activo del Irinotecan (un inhibidor de topoisomerasa 1), lo que permite la entrega de dosis altas, sitio específicas del citotóxico. Sacituzumab govitecan ha mostrado beneficio en SLP para pacientes con CMTN avanzado ${ }^{35}$.

Tabla 4. Estudios fase 3 con inhibidores de checkpoint inmunes, con resultados positivos en cáncer de mama triple negativo

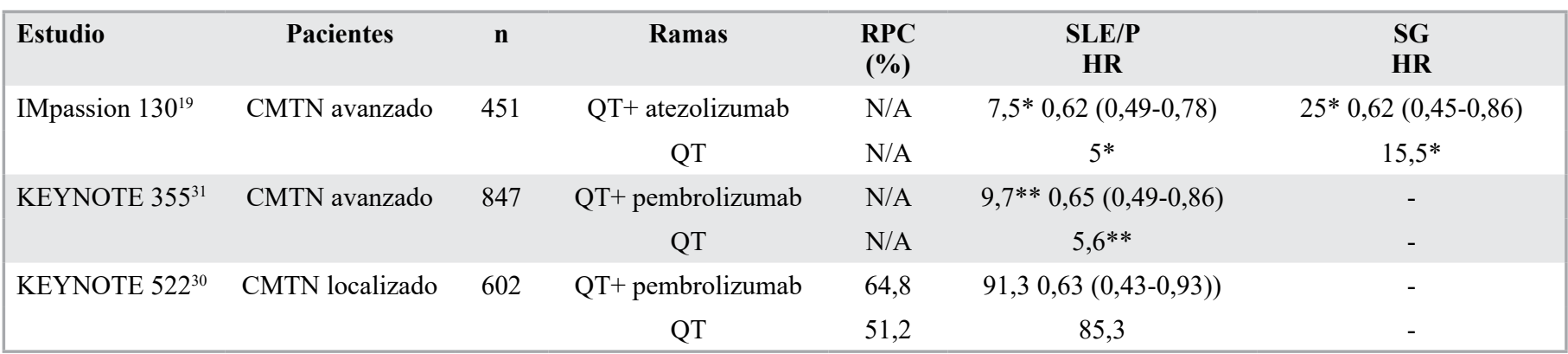

Abreviaciones: CMTN: cáncer de mama triple negativo; RPC: respuesta patológica completa; QT: quimioterapia; N/A: no aplica; SG: Sobrevida global; SLE: sobrevida libre de enfermedad (para CM localizado); SLP: sobrevida libre de progresión (para CM avanzado). *PDL1+ IC > 1\%. $* *$ PDL $1+$ CPS $>10$.

Tabla 5. Estudios fase 3 del uso de inhibidores PARP (poli-adenosina difosfato ribosa polimerasa) en cáncer de mama triple negativo

\begin{tabular}{|lccccc|}
\hline Estudio & Pacientes & n & Ramas & SLP & SG \\
OLYMPIAD $^{32}$ & CM avanzado BRCAm & 302 & Olaparib vs QT & 7 vs 4,2 meses & No diferencias \\
EMBRACA $^{33}$ & CM avanzado BRCAm & 431 & Talazoparib vs QT & 8,6 vs 5,6 meses & No diferencias \\
BRIGHTNESS $^{34}$ & CMTN, NA & 634 & $\begin{array}{c}\text { Veliparib + Paclitaxel + Carboplatino vs } \\
\text { Carboplatino + Paclitaxel }\end{array}$ & No evaluable & No evaluable \\
& & & &
\end{tabular}

Abreviaciones: CM: cáncer de mama; CMTN: cáncer de mama triple negativo; NA: neoadyuvancia; RPC: respuesta patológica completa; QT: quimioterapia; SLP: supervivencia libre de progresión SG: sobrevida global. 
Desafíos y oportunidades en cáncer de mama
triple negativo en Chile

América Latina es eminentemente heterogénea, compuesta por diferentes grupos étnicos, diversos patrones de migración históricos y grados variables de mestizaje; por lo tanto, con una fuerte diversidad genética que se suma a importantes diferencias ambientales y culturales. La mayoría de la información publicada de pacientes latinoamericanos con $\mathrm{CM}$ proviene de estudios realizados en población latina que vive en los Estados Unidos. Allí, registros del Surveillance, Epidemiology and End Results (SEER) han mostrado que las mujeres latinas tienen un mayor riesgo de mortalidad en comparación con las mujeres blancas no hispanas $(\mathrm{BNH})^{36}$. La mayor prevalencia de subtipos agresivos en mujeres latinas, particularmente $\mathrm{TN}$, además de factores culturales y socioeconómicos, estos últimos asociados a menor acceso a la atención médica, diagnóstico tardío, menor conocimiento de enfermedad y bajos niveles de educación, explicarían este fenómeno ${ }^{37}$. Datos del SEER también muestran que mujeres afroamericanas y latinas tenían proporcionalmente más CMTN (AA: OR = 2,0, 134 IC 95\%, 1,8-2,2; latinas: OR = 1,3 , IC 95\%, 1,2-1,5) en comparación con mujeres BNH. Recientemente, Rey Vargas et al..$^{38}$, en una revisión de la literatura de estudios publicados en los últimos 10 años (enero de 2008 - diciembre de 2018) referidos a CMTN en América Latina, buscó describir sus factores de riesgo asociados a este subtipo. Sus conclusiones se pueden resumir en que las mujeres latinas generalmente son diagnosticadas con $\mathrm{CM}$ a una edad más joven (56 vs 63 años para las mujeres $\mathrm{BNH}$ ) y en etapas más avanzadas que las mujeres BNH. Además, el bajo nivel educativo estaría asociado a un aumento de CMTN, tal vez relacionado con menor consumo de alimentos saludables, mayor sedentarismo y obesidad ${ }^{39}$. Sin embargo, como Rey Vargas concluye en su revisión, las inconsistencias entre los diferentes estudios epidemiológicos en mujeres latinas justifican el estudio centrado en los factores de riesgo específicos de CMTN para cada población ${ }^{38}$.

Nuestro grupo cuenta actualmente con una base de datos retrospectiva de $\mathrm{CM}$ que recopila datos clínicos, patológicos y de supervivencia. En esta describimos, según datos publicados ${ }^{5}$, que la pro- porción de CMTN en dos hospitales en Santiago, Chile, es cercana al $11 \%$, similar a la descrita en la población general en los Estados Unidos y menor a lo reportado en series de población latina ${ }^{37}$. Además, de manera similar a los datos de SEER, la proporción relativa de CMTN ha disminuido desde el año 1997; sin diferencias en la mediana de edad entre RE + y RE-. Tenemos un número menor de pacientes diagnosticados en etapa IV de novo (7\%), a pesar de un limitado diagnóstico en mujeres asintomáticas (por hallazgos en mamografía) en comparación con otros países; sólo el $17 \%$ vs más de $50 \%{ }^{40}$.

Estos datos sugieren que es relevante determinar características locales de mujeres con CMTN, pues difieren de lo reportado y extrapolado de datos obtenidos en otras poblaciones.

\section{Conclusiones}

Existen nuevas terapias moleculares efectivas para el CMTN. Ejemplo de ellas son los inhibidores de puntos de control inmune e inhibidores de la PARP; ellos requieren de la determinación de biomarcadores para su indicación, por lo tanto, es relevante entender la heterogeneidad de esta enfermedad, los mecanismos de acción de las terapias, resultados clínicos y criterios de selección.

Estas nuevas estrategias de diagnóstico y manejo no están ampliamente disponibles en Chile ni en América Latina. Existen escasos estudios sobre el CMTN en nuestra población, hecho que resalta la gran importancia de determinar y conocer las características locales de esta enfermedad para optimizar su manejo.

\section{Responsabilidades éticas}

Protección de personas y animales. Los autores declaran que para esta investigación no se han realizado experimentos en seres humanos ni en animales.

Confidencialidad de los datos. Los autores declaran que en este artículo no aparecen datos de pacientes.

Conflictos de interés: no hay. 


\section{Bibliografía}

1. Bray F, Ferlay J, Soerjomataram I, Siegel RL, Torre LA, Jemal A. Global cancer statistics 2018: GLOBOCAN estimates of incidence and mortality worldwide for 36 cancers in 185 countries. CA Cancer J Clin. 2018;68:394-424.

2. Perou CM, Sørlie T, Eisen MB, van de Rijn M, Jeffrey SS, Rees Ca, et al. Molecular portraits of human breast tumours. Nature. 2000;406:747-52.

3. Shim HJ, Kim SH, Kang BJ, Choi BG, Kim HS, Cha ES, et al. Breast cancer recurrence according to molecular subtype. Asian Pac J Cancer Prev. 2014;15:5539-44.

4. Harbeck N, Gnant M. Breast cancer Lancet. 2017;389(10074):1134-50.

5. Maiz C, Silva F, Domínguez F, Galindo H, Camus M, León A, et al. Mammography correlates to better survival rates in breast cancer patients: a 20-year experience in a University health institution. Ecancermedicalscience. 2020 Jan;14.

6. Turkoz FP, Solak M, Petekkaya I, Keskin O, Kertmen N, Sarici F, et al. Association between common risk factors and molecular subtypes in breast cancer patients. Breast 2013;22:344-50.

7. Yang XR, Chang-Claude J, Goode EL, Couch FJ, Nevanlinna H, Milne RL, et al. Associations of breast cancer risk factors with tumor subtypes: a pooled analysis from the Breast Cancer Association Consortium studies. J Natl Cancer Inst. 2011;103:250-63.

8. Schmadeka R, Harmon BE, Singh M. Triple-negative breast carcinoma: current and emerging concepts. Am J Clin Pathol. 2014;141:462-77.

9. Dai X, Li T, Bai Z, Yang Y, Liu X, Zhan $\mathrm{J}$, et al. Breast cancer intrinsic subtype classification, clinical use and future trends. Am J Cancer Res. 2015;5:2929-43.

10. Garmpis N, Damaskos C, Garmpi A, Nikolettos K, Dimitroulis D, Diamantis E, et al. Molecular Classification and Future Therapeutic Challenges of Triple-negative Breast Cancer. In Vivo (Brooklyn). 2020;34:1715-27.

11. Dai X, Xiang L, Li T, Bai Z. Cancer Hallmarks, Biomarkers and Breast Cancer Molecular Subtypes. J Cancer 2016;7:1281-94.

12. Cetin I, Topcul M. Triple negative breast cancer. Asian Pac J Cancer Prev. 2014;15:2427-31.

13. Masuda N, Lee SJ, Ohtani S, Im YH, Lee ES, Yokota I, et al. Adjuvant capecitabine for breast cancer after preoperative chemotherapy. N Engl J Med. 2017;376:2147-59. doi: 10.1056/ NEJMoa1612645.

14. Lluch A, Barrios CH, Torrecillas L, RuizBorrego M, Bines J, Segalla J, et al. Phase III Trial of Adjuvant Capecitabine After Standard Neo-/Adjuvant Chemotherapy in Patients With Early Triple-Negative Breast Cancer (GEICAM/2003-11 CIBOMA/2004-01). J Clin Oncol. 2020;38:203-13.

15. Xu M, Yuan Y, Yan P, Jiang J, Ma P, Niu X, et al. Prognostic Significance of Androgen Receptor Expression in Triple Negative Breast Cancer: A Systematic Review and Meta-Analysis. Clin Breast Cancer. 2020;20:e385-e396. doi: 10.1016/j.clbc.2020.01.002. Epub 2020 Jan 18.

16. Thakkar A, Wang B, Picon-Ruiz M, Buchwald P, Ince TA. Vitamin D and androgen receptor-targeted therapy for triple-negative breast cancer. Breast Cancer Res Treat. 2016;157:77-90.

17. Brufsky A, Valero V, Tiangco B, Dakhil S, Brize A, Rugo HS, et al. Second-line bevacizumab-containing therapy in patients with triple-negative breast cancer: Subgroup analysis of the RIBBON-2 trial. Breast Cancer Res Treat [Internet]. 2012 Jun 14 [cited 2020 Jul 5];133(3):106775. Available from: http://link.springer. com/10.1007/s10549-012-2008-6

18. Barroso-Sousa R, Tolaney SM. Clinical Development of PD-1/PD-L1 Inhibitors in Breast Cancer: Still a Long Way to Go. Vol. 21, Current Treatment Options in Oncology. Springer; 2020.

19. Schmid P, Chui SY, Emens LA. Atezolizumab and Nab-Paclitaxel in Advanced Triple-Negative Breast Cancer. N Engl J Med. 2019;380:985-8.

20. Jara L, Morales S, De Mayo T, GonzálezHormazábal P, Carrasco V, Godoy R. Mutations in BRCA1, BRCA2 and other breast and ovarian cancer susceptibility genes in Central and South American populations. Biol Res. 2017;50:35. doi: 10.1186/s40659-017-0139-2.

21. Lurie RH, Anderson BO, Abraham J, Aft $\mathrm{R}$, Agnese D, Allison KH, et al. NCCN
Guidelines Version 4.2020 Breast Cancer.

22. Loibl S, Weber KE, Timms KM, Elkin EP, Hahnen E, Fasching PA, et al. Survival analysis of carboplatin added to an anthracycline/taxane-based neoadjuvant chemotherapy and HRD score as predictor of response-final results from GeparSixto. Ann Oncol. 2018;29(:2341-7. doi: 10.1093/annonc/mdy460.

23. Sikov W, Berry D, Perou C, Singh B, Cirrincione C, Tolaney S, et al. Abstract S2-05: Event-free and overall survival following neoadjuvant weekly paclitaxel and dose-dense $\mathrm{AC}+/$ - carboplatin and/ or bevacizumab in triple-negative breast cancer: Outcomes from CALGB 40603 (Alliance). In 2016

24. Cortázar P, Zhang L, Untch M, Mehta $\mathrm{K}$, Costantino JP, Wolmark N, et al. Pathological complete response and longterm clinical benefit in breast cancer: The CTNeoBC pooled analysis. Lancet 2014;384(9938):164-72. doi: 10.1016/ S0140-6736(13)62422-8. Epub 2014 Feb 14.

25. Rastogi P, Geyer CE, Mamounas EP, De Michele A. Drug Development: Neoadjuvant Opportunities in Breast Cancer. Am Soc Clin Oncol Educ B. 2013;33:73-9

26. Sánchez C, Camus M, Medina L, Oddo D, Artigas R, Sepúlveda APAP, et al. ClinicoPathologic Subtypes of Breast Cancer Primary Tumors Are Related to Prognosis after Recurrence. Asian Pac J Cancer Prev. 2016;17:5081-6.

27. Tutt A, Tovey H, Chon M, Cheang U, Kernaghan S, Kilburn L, et al. A randomised phase III trial of carboplatin compared with docetaxel in BRCA1/2 mutated and pre-specified triple negative breast cancer "BRCAness" subgroups: the TNT Trial Europe PMC Funders Group. Nat Med. 2018;24:628-37.

28. Barroso-Sousa R, Tolaney SM. Clinical Development of PD-1/PD-L1 Inhibitors in Breast Cancer: Still a Long Way to Go. Options in Oncol. 2020;21:59.

29. Loibl S, O'Shaughnessy J, Untch M, Sikov WM, Rugo HS, McKee MD, et al. Addition of the PARP inhibitor veliparib plus carboplatin or carboplatin alone to standard neoadjuvant chemotherapy in triple-negative breast cancer (BrighTNess): a randomised, phase 3 trial. Lancet Oncol. 2018;19:497-509. 
30. Schmid P, Cortés J, Pusztai L, McArthur H, Kümmel S, Bergh J, et al. Pembrolizumab for early triple-negative breast cancer.

N Engl J Med. 2020;382:810-21. doi: 10.1056/NEJMoa1910549.

31. Cortés J, Cescon DW, Rugo HS, Nowecki Z, Im S-A, Yusof MM, et al. KEYNOTE-355: Randomized, doubleblind, phase III study of pembrolizumab + chemotherapy versus placebo + chemotherapy for previously untreated locally recurrent inoperable or metastatic triple-negative breast cancer. J Clin Oncol. 2020;38(15_suppl):1000-1000.

32. Robson M, Im S-A, Senkus E, Xu B, Domchek SM, Masuda N, et al. Olaparib for Metastatic Breast Cancer in Patients with a Germline BRCA Mutation. N Engl J Med. 2017;377:523-33.
33. Litton JK, Rugo HS, Ettl J, Hurvitz SA, Gonçalves A, Lee K-H, et al. Talazoparib in Patients with Advanced Breast Cancer and a Germline BRCA Mutation. N Engl J Med. 2018;379:753-63.

34. Loibl S, O'Shaughnessy J, Untch M, Sikov WM, Rugo HS, McKee MD, et al. Addition of the PARP inhibitor veliparib plus carboplatin or carboplatin alone to standard neoadjuvant chemotherapy in triple-negative breast cancer (BrighTNess): a randomised, phase 3 trial. Lancet Oncol. 2018;19:497-509. doi: 10.1016/S14702045(18)30111-6.

35. Bardia A, Mayer IA, Vahdat LT, Tolaney SM, Isakoff SJ, Diamond JR, et al. Sacituzumab Govitecan-hziy in Refractory Metastatic Triple-Negative Breast Cancer. N Engl J Med. 2019;380:741-51.

36. DeSantis CE, Ma J, Gaudet MM, Newman
LA, Miller KD, Goding Sauer A, et al. Breast cancer statistics, 2019. CA Cancer J Clin. 2019;69:438-51.

37. Cazap E. Breast Cancer in Latin America: A Map of the Disease in the Region. Am Soc Clin Oncol Educ B. 2018;38:451-6.

38. Rey-Vargas L, Sanabria-Salas MC, Fejerman L, Serrano-Gómez SJ. Risk Factors for Triple-Negative Breast Cancer among Latina Women. Cancer Epidemiol Biomarkers Prev. 2019;28:1771-83.

39. Copson ER, Cutress RI, Maishman T, Eccles BK, Gerty S, Stanton L, et al. Obesity and the outcome of young breast cancer patients in the UK: the POSH study. Ann Oncol. 2015;26:101-12.

40. Li JJ, Shao ZM. Mammography screening in less developed countries. Vol. 4, SpringerPlus. SpringerOpen; 2015. p. 615. 